\title{
Carottage profond sur le glacier du Lys (Monte Rosa) : résultats préliminaires
}

\author{
Deep coring on the Lys glacier (Monte Rosa) : first results
}

par G. Rossi - ENEL S.p.A. Divisione produzione - Ingegneria Civile Idraulica - Venezia

A. Novo - ENEL S.p.A. DSR CRAM Milano,

V. Maggi, G. Orombelli - Universita di Milano, Dipart. Scienze Ambiente e Territorio

C. Smiraglia - Universita di Milano, Dipart. Scienze della Terra

After a deep coring in the Lys glacier and ice density measurement, stratigraphy plotting and isotopic radiometry analyses were carried out. They led to the reconstitution of some aspects of the recent past climate in the Alps.

\section{I INTRODUCTION}

Après les forages européens effectués par des chercheurs suisses et allemands sur le plateau du Colle Gnifetti (Monte Rosa à $4450 \mathrm{~m}$ d'altitude) et par des chercheurs français au Col du Dôme (Mont Blanc à $4250 \mathrm{~m}$ d'altitude), une équipe italienne a effectué en 1995 un premier forage au Colle Gnifetti (sur le Grenzegletscer en territoire helvétique) permettant de rejoindre une profondeur de $24 \mathrm{~m}$. Au cours des premiers jours de juin 1996 a été également effectué un forage complet sur le versant italien du monte Rosa, au Colle del Lys à une altitude de $4240 \mathrm{~m}$.

Cette relation en décrit les opérations et les résultats préliminaires obtenus.

\section{II — OBJECTIFS ET MÉTHODES}

Le fait de disposer d'une carotte de glace extraite d'un glacier alpin permet d'entreprendre une recherche orientée vers les objectifs suivants :

- l'étude de l'histoire du climat et de l'atmosphère de la dernière moitié du siècle ;

- l'évaluation de l'impact anthropogénétique sur le milieu alpin dans le même laps de temps.

Ces objectifs peuvent être rejoints à travers les phases suivantes :

- analyses permettant d'identifier et de quantifier le contenu des agents contaminants aux diverses profondeurs des couches de glace,

- identification de la relation entre l'accumulation de masse dans la glace et la chronologie des phénomènes de dépôt identifiés,
- interprétation des informations relatives au contenu des dépôts et à leur chronologie afin d'obtenir des informations sur la dynamique des événements les ayant déterminés,

- validation des résultats en termes de représentativité spatiale et temporelle.

Le programme des recherches prévoit donc des mesures et des observations sur le forage ainsi que des recherches sur les échantillons, sur place comme en phase de laboratoire. Les recherches en laboratoire doivent permettre de définir les points suivants :

- conductibilité électrique en phase solide (ECM) comme en phase liquide,

- analyse chimique quantitative des ions principaux comme $\mathrm{SO}_{4}, \mathrm{NO}_{3}, \mathrm{Cl}, \mathrm{HCO}_{3}, \mathrm{Na}, \mathrm{K}, \mathrm{NH}_{4}, \mathrm{Ca}, \mathrm{Mg}$, acidité, détermination des isotopes stables $\left({ }^{18} \mathrm{O}\right.$ e $\left.\mathrm{D}\right)$, détermination des isotopes radioactifs, ${ }^{3} \mathrm{H},{ }^{210} \mathrm{~Pb}$ et bêta totaux, analyse des poussières insolubles, propriétés physiques de la glace.

C'est également grâce à la collaboration ainsi qu'à l'expérience fournie par les collègues du LGGE de Grenoble et de l'Université de Heidelberg [1] que le développement de la recherche dans son état actuel a été rendu possible.

\section{III $\square$ DESCRIPTION DES SITES}

Le forage a été effectué sur le versant italien du monte Rosa, au Colle del Lys à une altitude de $4240 \mathrm{~m}$, durant les premiers jours de juin 1996. Le site [DATUM ED50 N5085865.05 E411651.40 altitude du géoïde $4241,70 \mathrm{~m}$ ] est localisé au sommet du bassin collecteur du glacier du même nom le long de l'alignement entre la crête du Lyskamm et la Punta Ludovica (fig. 1), à l'intérieur d'une zone intéressée principalement par des masses d'air provenant du sud, carac- 
térisée par un régime de précipitations qui alimentent les dépôts s'accumulant principalement en période estivale. Ces dépôts, qui s'accumulent à une vitesse équivalent à une moyenne de 1,5 - 2 mètres d'eau par an, sont capables de fournir des informations à haute résolution temporelle, mais, étant donné que la masse de glace ne peut rejoindre une épaisseur totale supérieure à 100 mètres, le potentiel d'horizon temporel est sensiblement inférieur à un siècle.

\section{IV — RECHERCHES PRÉLIMINAIRES}

Les glaciers des versant italiens du Monte Rosa ont été l'objet d'une longue tradition d'études glaciologiques dont les origines remontent aux premières années de ce siècle, principalement par l'œuvre de Monterin [2]. Ces études ont été interrompues dans la période de l'après-guerre jusqu'au début des années 90 où ont été mises en place une série d'initiatives dont la première était un projet quinquennal (1991-95), d'échantillonage et d'analyse du contenu des dépôts neigeux de la fin du printemps, faisant partie d'un vaste projet européen EUROTRAC/ALPTRAC, concernant également quelques sites du versant suisse du Monte Rosa. Ces recherches ayant été menées par les Centres de Recherche CRIS et CRAM de l'ENEL S.p.A., comportaient également le monitorage des principaux paramètres météorologiques et énergétiques d'une année entière, avec l'installation d'une station météorologique au Col Vincent, à $4088 \mathrm{~m}$ d'altitude [3].

A la même période, grâce à une collaboration entre l'ENEL S.p.A. et le Comité Glaciologique Italien, a été mis en place un programme d'évaluation du bilan de masse du glacier à travers des mesures de surface et des mesures hydrauliques [4].

Au cours des années 1995 et 1996 ont été effectués respectivement : un relevé aérophotogrammétrique de détail de l'ensemble du système glaciaire et sa restitution cartographique numérique et deux campagnes de prospection géophysique à l'aide d'un radar afin de déterminer l'épaisseur de la masse glaciaire. Les résultats preliminaires de cette enquête indiquent une épaisseur de 70-80 m de glace dans la zone du forage et des épaisseurs de $80-100 \mathrm{~m}$ dans la zone du Col Vincent.

\section{I LE FORAGE AU COLLE DEL LYS}

L'expédition a été mise en place à travers une collaboration entre les Centres de Recherche CRIS et CRAM de l'ENEL S.p.A., le Département de Sciences de l'Environment et du Territoire (DISAT) et le Département de Sciences de la Terre (DIST) de l'Université des Etudes de Milan, le Département de Sciences de la Terre de l'Université de Trieste et le Programme National Recherches en Antarctique (PNRA) ayant apporté l'équipement logistique et technique.

Le forage a été effectué à l'aide d'une foreuse de fabrication suisse, de type rotatif, n'endommageant pas le matériel extrait, manœuvrée à l'aide d'un treuil capable de rejoindre la profondeur maximum de $150 \mathrm{~m}$. La fraise fonctionnant de manière discontinue était capable de travailler des carottes d'une longueur de $50-90 \mathrm{~cm}$ et d'un diamètre de $10 \mathrm{~cm}$.

La durée du forage a été de cinq jours à la fin desquels une profondeur de $80 \mathrm{~m}$ a été atteinte. La progression, en termes de vitesse d'avancement du forage, a été régulière avec des valeurs journalières proches de la valeur moyenne sur l'ensemble de la période.
Le succès du forage, mesuré en fonction du pourcentage de la longueur des carottes utilisables sur la longueur totale du trou, a été très important, étant environ de $98 \%$ sur les premiers 60 mètres.

Dans les carottes extraites à une profondeur de 77-80 m, ont été observés des petits débris de roches, de quelques millimètres de diamètre, qui en général indiquent que la glace basale a été rejointe.

Le forage a donc été arrêté afin d'éviter d'endommager le carottier qui n'aurait pu affronter l'impact avec le lit de roche. La valeur de la profondeur atteinte concordait parfaitement avec le résultat de la prospection radar ayant donné pour les alentours du point de forage une valeur de 80 mètres.

\section{VI — RÉSULTATS PRÉLIMINAIRES DES MESURES}

\section{- 6.1 Mesures physiques dans le trou}

A la fin du forage une sonde de température a été laissée à l'intérieur du trou avec trois capteurs disposés respectivement à -80 mètres de hauteur du fond, à environ la moitié ( -50 mètres) et un autre à -15 mètres. La première mesure significative a été effectuée après environ cinq mois durant lesquels s'était accumulée une couche de 5 mètres de neige par rapport à la période du forage. Les mesures à l'intérieur du trou ont été enregistrées en continu pendant environ une heure et ont fourni les valeurs reportées sur le tableau 1, alors que la mesure à -2 mètres a été enregistrée en continu de la station météorologique. Le gradient de l'intervalle compris entre $-15 \mathrm{~m}$ et $-50 \mathrm{~m}$ de profondeur est de $-0,13^{\circ} \mathrm{C} / \mathrm{m}$ ). Même si le nombre réduit de points de mesure ne permet pas d'évaluer ce processus de façon plus précise, il est toutefois possible de constater que le gradient mantient toujours son signe négatif lorsque la profondeur augmente, contrairement à ce qui avait pu être noté sur le site proche du Colle Gnifetti [5].

Les résultats confirment d'autre part que les températures mesurées dans la glace du secteur le plus élevé du bassin collecteur au Colle del Lys présentent des valeurs typiques des "glaciers froids".

\subsection{Mesures physiques sur les carottes}

\subsubsection{Mesures de densité}

Les résultats des mesures de densité du glacier varient d'un minimum de $0,32 \mathrm{~g} / \mathrm{cm}^{3}$ à la surface jusqu'à un maximum de $0,91 \mathrm{~g} / \mathrm{cm}^{3}$ dans les parties les plus profondes ; la transition névé-glace se produit aux alentours d'une profondeur de $-40 \mathrm{~m}$. Le rapport entre densité et profondeur est très net mais il est toutefois possible d'observer des valeurs individuelles ponctuelles qui diffèrent même sensiblement de la tendence à l'augmentation habituelle (exception faite des erreurs de mesurage dues à des irrégularités géométriques du fragment de carotte). Ces irrégularités peuvent être expliquées par la présence de lentilles et de couches de glaces de regel qui augment les valeurs de la densité même ; il est en effet possible d'observer, sur la fig. 2, que les fluctuations majeures de densité sont situées dans les 20 premiers mètres de carotte, au niveau des concentrations majeures de lentilles de glace.

Les variations de gradient peuvent au contraire être mises en rapport avec les diverses phases du processus de transformation de la neige en glace. Jusqu'à 10 mètres environ, avec un gradient de $0,028 \mathrm{~g} / \mathrm{cm}^{3} / \mathrm{m}^{-1}$ se produit un premier pro- 


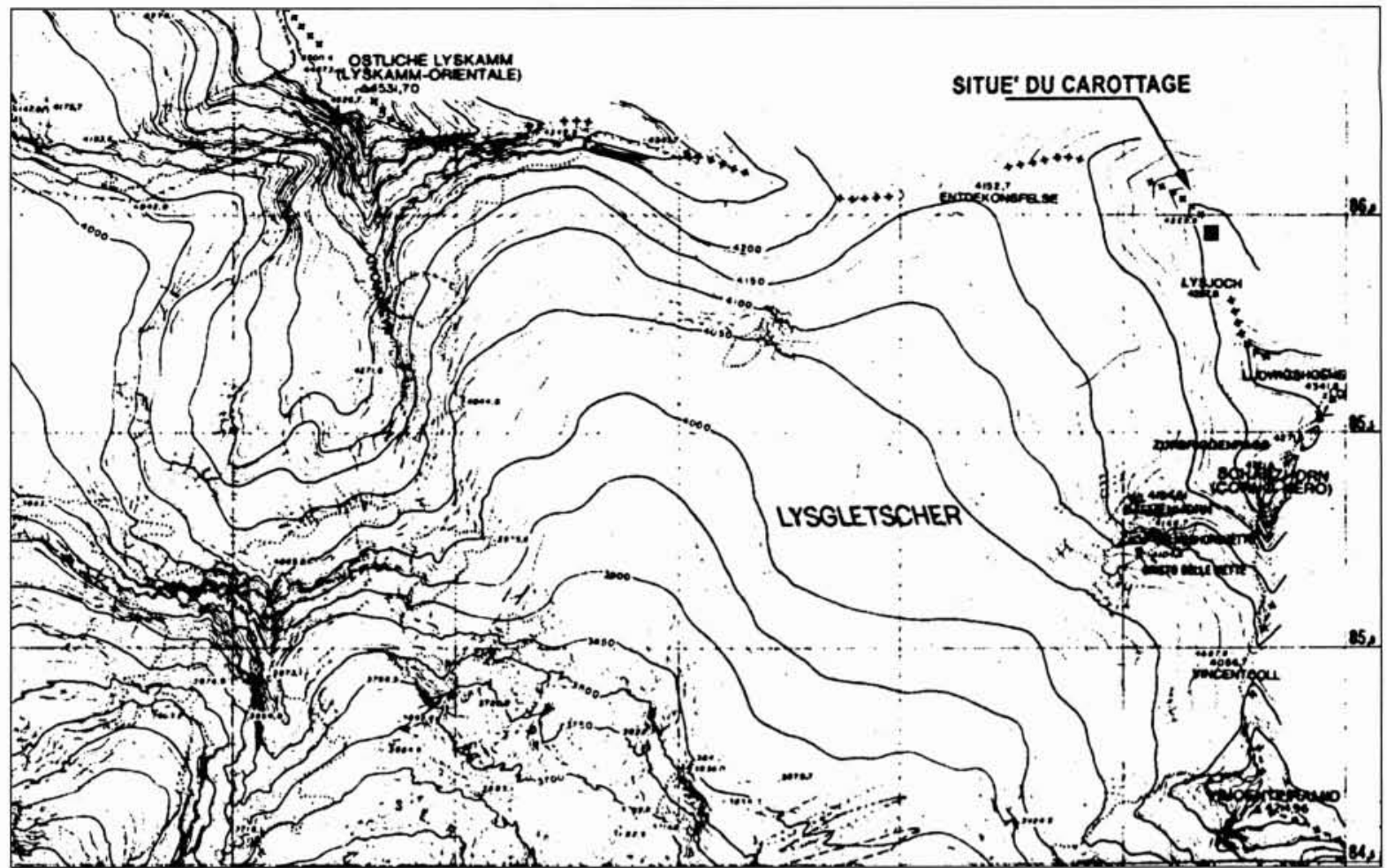

1. Localisation du site de carottage du Col du Lys.

cessus de formation de névé à travers le compactage et le damage des cristaux qui conduit à une densité d'environ $0,55 \mathrm{~g} / \mathrm{cm}^{3}$. De 10 à $50 \mathrm{~m}$ environ, l'augmentation de densité suit un gradient de $0,013 \mathrm{~g} / \mathrm{cm}^{3} / \mathrm{m}^{-1}$ qui conduit à la transformation complète en névé et à la transition névé-glace, avec une densité de $0,85 \mathrm{~g} / \mathrm{cm}^{3}$ quand le processus de "sealing off" est terminé. Entre 40 et $70 \mathrm{~m}$, la densité n'augmente que légèrement (le gradient est inférieur à $0,003 \mathrm{~g} / \mathrm{cm}^{3} / \mathrm{m}^{-1}$ ) et dépasse également $0,91 \mathrm{~g} / \mathrm{cm}^{3}$. Il s'agit de la phase de compression des bulles d'air enfermées dans les cristaux de glace. En-dessous de $70 \mathrm{~m}$, la mauvaise qualité de la carotte, brisée en nombreux fragments, n'a plus permis de mesurer la densité.

\subsubsection{Stratigraphie visible}

La stratigraphie visible et a été tracée directement dans le laboratoire froid et révèle la présence de lentilles et de couches de glace de regel. La distribution des lentilles de glace pourrait également fournir quelques indications chronologiques ; les concentrations les plus importantes de lentilles et de couches de glace devraient en effet correspondre aux étés particulièrement chauds, alors que les étés très froids ne devraient pas conduire à la formation de lentilles de glace. Sur les 20 premiers mètres de carotte peuvent être notées des épaisseurs importantes ainsi que la présence plus fréquente de lentilles de glace par rapport à la partie inférieure, ce qui pourrait laisser penser à une tendance récente d'augmentation des températures estivales.

Il est d'autre part possible d'observer sur la carotte des couches marquées de dépôts secs ayant pour origine le transport éolien de poussières provenant des régions désertiques africaines. Le plus significatif de ces phénomènes a été trouvé à environ $55 \mathrm{~m}$ de profondeur et pourrait être attribué à celui particulièrement intense s'étant produit en 1977. Si cela devait être confirmé, l'accumulation nette en équivalent d'eau pour la période 1977-96 pourrait être environ de $1600 \mathrm{~mm} / \mathrm{an}$.

\subsection{Analyses de radiométrie isotopique}

A l'état actuel des analyses, seules sont disponibles les premières données concernant la concentration de Tritium $3 \mathrm{H}$, isotope se produisant au cours des réactions thermonucléaires. La courbe des concentrations mesurées de cet isotope est reportée sur le graphique de la figure 2 et met en évidence un pic net de concentration localisé à un niveau équivalent en eau d'environ $55 \mathrm{~m}$ de profondeur. Si ce signal est attribué à la dernière série de tests thermonucléaires effectués en 1963 en atmosphère, on obtient une accumulation annuelle moyenne de $1700 \mathrm{~mm} / \mathrm{an}$ en équivalent d'eau, valeur qui confirmerait ce qui a été déduit précédemment. Il n'est pas possible cependant d'observer des fluctuations périodiques de la concentration permettant de déterminer un mouvement saisonnier.

\section{VII — CONCLUSIONS}

Le fait de disposer d'une carotte provenant d'un forage profond du glacier du Lys permet d'effectuer une série de recherches permettant de reconstruire l'évolution de certains aspects climatiques et ambiants d'un passé récent sur le territoire alpin ; avant toute chose, le site de provenance a présenté les conditions de température de glace nécessaires à la préservation des informations. 
Les premiers résultats des analyses sur les carottes de névé et de glace confirment la valeur importante de l'accumulation nette annuelle (1600-1700 mm/an), valeur obtenue en identifiant des phénomènes singuliers sur la carotte pouvant être associés à des dates certaines (poussières sahariennes, pic de concentration de Tritium). La transformation du névé en glace se produit à une profondeur d'environ $40 \mathrm{~m}$.

Les recherches actuellement en cours ont pour objectif de mettre en évidence des variations récentes de la composition chimique des composés transportés par la circulation atmosphérique, afin de confirmer surtout les effets que les politiques de diminution des émissions des installations produisant de l'énergie électrique à l'aide de combustibles à faible contenu en soufre, ont eu sur l'atmosphère, effets qui par ailleurs ont déjà été mis en évidence par d'autres recherches.

Un intérêt particulier subsiste d'autre part concernant les relations existant entre précipitations, température, accumulation de glace et gestion des réserves hydriques.

\section{Références}

[1] WAGENBACH D. (1989) : "Environmental Records in Alpine Glaciers", The Environmental Record in Glaciers and Ice Sheeets, H. Oeschger \& C.C. Langway Eds.

[2] MONTERIN U, (1991) : "I ghiacciai del Monte Rosa", Raccolta di scritti di Umbero Monterin, Vol. III, a cura di A.V. Cerutti, Librairie Valdotaine, Aosta

[3] NOVO A., ROSSI G.C. (1997) : Relazione conclusiva delle campagne ALPTRAC (1991 - 95) in press

[4] ROSSI G., MOTTA M. (1997) : "Bilancio di massa dei ghiacciai del Monte Rosa 1991-1995", in press.

[5] HEBERL1 V., SCHIMID W., WAGENBACH D. (1988): "On the geometry, flow and age of firn and ice at the Colle Gnifetti core drilling site (Monte Rosa, Swiss Alps)", Zeitschr. für Gletsch. und Glazial.
Tab. 1 - Courbe des températures mesurées dans le gouffre del glace le 19/12/96

\begin{tabular}{|c|c|}
\hline Profondeur & Température $\left({ }^{\circ} \mathrm{C}\right)$ \\
\hline$-2 \mathrm{~m}$ & $-11,0$ \\
$-15 \mathrm{~m}$ & $-6,0$ \\
$-50 \mathrm{~m}$ & $-10,8$ \\
$-80 \mathrm{~m}$ & $-11,2$ \\
\hline
\end{tabular}

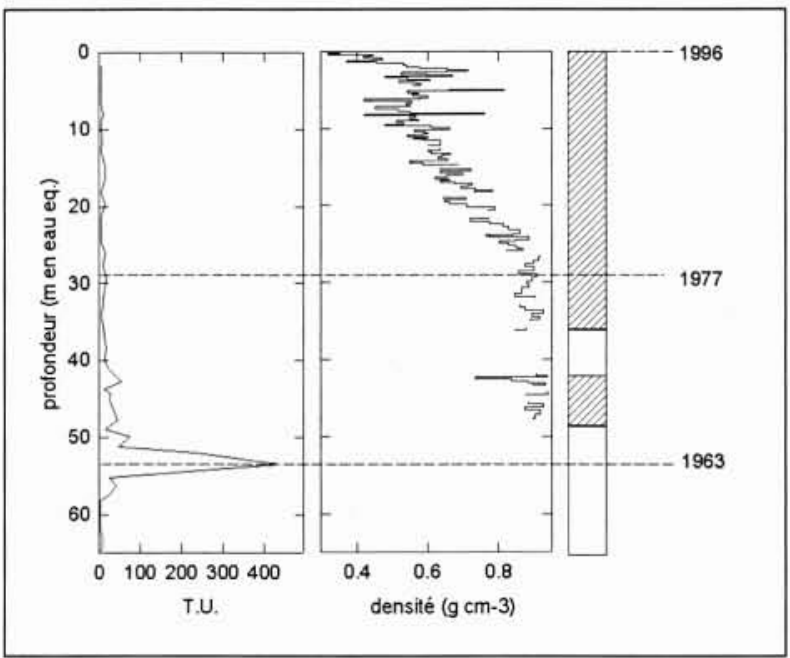

2. Stratigraphie de la densité et de la concentration de Tritium 